\title{
Connected Components of the Hurwitz Space for the Symmetric Group of Degree 7
}

\author{
Haval M. Mohammed Salih
}

Soran University, Faculty of Science, Mathematics Department-Kawa St, Soran, Erbil, Iraq; University of Raparian, College of Basic Education, Department of Mathematic, Ranya, Kurdistan Region of Iraq. Email: haval.mahammed@soran.edu.iq.

\begin{abstract}
The Hurwitz space $\mathcal{H}_{\mathrm{r}}^{\mathrm{in}}(\mathrm{G})$ is the space of genus $\mathrm{g}=0$ covers of the Riemann sphere $\mathbb{P}^{1}$ with $\mathrm{r}$ branch points and the monodromy group $G$. Let $G$ be the symmetric group $S_{7}$. In this paper, we enumerate the connected components of $\mathcal{H}_{\mathrm{r}}^{\text {in }}\left(\mathrm{S}_{7}\right)$. Our approach uses computational tools, relying on the computer algebra system GAP and the MAPCLASS package, to find the connected components of $\mathcal{H}_{\mathrm{r}}^{\mathrm{in}}\left(\mathrm{S}_{7}\right)$. This work gives us the complete classification of primitive genus zero symmetric group of degree seven.
\end{abstract}

Keywords: Monodromy Groups; Braid Orbits; Connected Components.

$$
\text { المكونات المتصلة لفضاء هيوريتز للزمرة تناظرية من الارجة } 7
$$

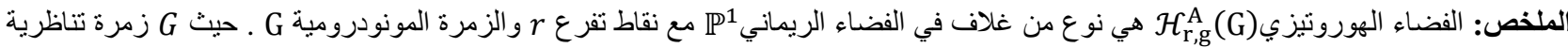

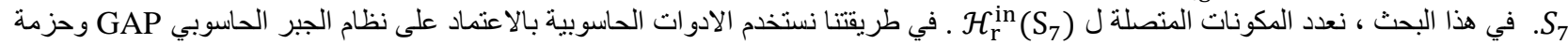

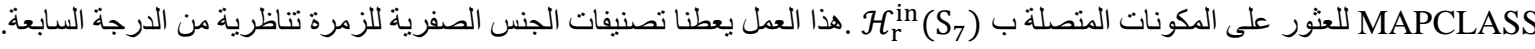

$$
\text { الكلمات المفتاحية: زمرة المنودرومية، مدار ات بريد و المكونات المتصلة. }
$$

\section{Introduction}

et us start this section by the following definition:

A primitive genus $g$ system is a triple $\left(G, \Omega,\left(x_{1}, \ldots, x_{r}\right)\right)$ where $\Omega$ is a finite set of size $n$ and $G$ is a primitive subgroup of $S_{n}$ such that

$$
\begin{gathered}
G=<x_{1}, \ldots, x_{r}> \\
\prod_{i=1}^{r} x_{i}=1 \\
2(n+g-1)=\sum_{i=1}^{r} \text { ind } x_{i}
\end{gathered}
$$

where $x_{i} \in G \backslash\{1\}$. For $x \in G$ define ind $x=n-\operatorname{orb}(x)$, Fix $x=\{w \in \Omega \mid x w=w\}, f(x)=\mid$ Fix $x \mid$ and orb $(x)=$ $\frac{1}{d} \sum_{i=0}^{d-1} f\left(x^{i}\right)$, where $d$ is the order of $x$ in $S_{n}$. These conditions (1), (2) and (3) are equivalent to the existence of the branched cover $\mu: X \rightarrow \mathbb{P}^{1}$, where $X$ is a Riemann surface of genus $g$. The number of holes is called the genus, and $\mu$ is a meromorphic function where $\mathbb{P}^{1}=\mathbb{C U}\{\infty\}$ is the Riemann sphere.

Let $C_{i}$ be a non-trivial conjugacy class of $x_{i}$. Then the set $C=\left\{C_{1}, \ldots, C_{r}\right\}$ in $G$ is called the ramification type of the cover $\mu$. Note that the trivial conjugacy class contains only the identity element.

In this paper, we classify primitive genus 0 systems for $S_{7}$. It is clear that there are seven primitive groups of degree 7. In [1], we classified all those groups except $S_{7}$. Now we are going to classify the group $S_{7}$ by using the computer algebra system GAP. All together give the complete classification of primitive genus 0 groups of degree 7 .

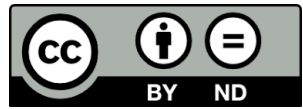


Braid orbits can be interpreted as saying interesting things about components of the moduli space of curves $\mathcal{M}_{g}$ [3] and equivalence classes of branched covers of the Riemann sphere $\mathbb{P}^{1}$.

The full details of the following results and concepts can be found in [1-11].

Let $C_{1}, \ldots, C_{r}$ be non-trivial conjugacy classes of a finite group $G$. The set of generating systems $\left(x_{1}, \ldots, x_{r}\right)$ of $G$ with $x_{1} \ldots x_{r}=1$ and such that there is a permutation $\pi \in S_{r}$ with $x_{i} \in S_{\pi(i)}$ for $i=1, \ldots, r$ is called a Nielsen class and denoted by $\mathcal{N}(\mathrm{C})$, where $C=\left(C_{1}, \ldots, C_{r}\right)$.

Each Nielsen class is the disjoint union of braid orbits, which are defined as the smallest subsets of the Nielsen class closed under the braid operations

$$
\left(x_{1}, \ldots, x_{r}\right)^{Q_{i}}=\left(x_{1}, \ldots, x_{i+1}, x_{i+1}^{-1} x_{i} x_{i+1}, \ldots, x_{r}\right)
$$

for $i=1, \ldots, r$.

We denote by $\mathrm{O}_{\mathrm{r}}$, the space of subsets of $\mathbb{C}$ of cardinality $\mathrm{r}$.

Definition 1.1 Let $B \in O_{r}$ and $b_{0} \in \mathbb{P}^{1} \backslash B$. We call a map $\varphi: \pi_{1}\left(\mathbb{P}^{1} \backslash B, b_{0}\right) \rightarrow G$ admissible if it is a surjective homomorphsim, and $\varphi\left(\theta_{b}\right) \neq 1$ for each $b \in B$. Here $\theta_{b}$ is the conjugacy class of $\pi_{1}\left(\mathbb{P}^{1} \backslash B, b_{0}\right)$.

Definition 1.2 Let $B \in O_{r}$ and $\varphi: \pi_{1}\left(\mathbb{P}^{1} \backslash B, \infty\right) \rightarrow G$ be admissible. Then we say that two pairs $(\mathrm{B}, \varphi)$ and $(\overline{\mathrm{B}}, \bar{\varphi})$ are A-equivalent if and only if $B=\bar{B}$ and $\bar{\varphi}=a \circ \varphi$ for some a $\in \mathrm{A}$. Let $[\mathrm{B}, \varphi]_{\mathrm{A}}$ denote the A-equivalence class of $(B, \varphi)$. The set of equivalence classes $[B, \varphi]_{A}$ is denoted by $\mathcal{H}_{r}^{A}(G)$ and is called the Hurwitz space of $G$-covers.

Lemma 1.3 Let $C$ be a fixed ramifcation type in $G$, and the subset $\mathcal{H}_{r}^{\text {in }}(C)$ of $\mathcal{H}_{r}^{\text {in }}(G)$ consist of all $[B, \varnothing]_{A}$ with $B=\left\{b_{1}, \ldots, b_{r}\right\}, \emptyset: \pi_{1}\left(\mathbb{P}^{1} \backslash B, \infty\right) \rightarrow G$ and $\emptyset\left(\theta_{b_{i}}\right) \in C_{i}$ for $\mathrm{i}=1, \ldots, \mathrm{r}$. Then $\mathcal{H}_{r}^{A}(C)$ is a union of connected components in $\mathcal{H}_{\mathrm{r}}^{\mathrm{A}}(\mathrm{G})$. Under the bijection from Lemma 2.2, the fiber in $\mathcal{H}_{r}^{A}(C)$ over $\mathrm{B}_{0}$ corresponds the set $\mathcal{N}^{\mathrm{A}}(\mathrm{C})$. This yields a one to one correspondence between components of $\mathcal{H}_{r}^{A}(C)$ and the braid orbits on $\mathcal{N}^{A}(C)$. In particular, $\mathcal{H}_{r}^{i n}(C)$ is connected if and only if there is only one braid orbit.

Proof. For a proof see [2].

\section{Computing Indexes and Labeling Conjugacy Classes}

In this paper, we discuss two methods for computing index as follows:

\section{Method one (Via Fixed Points)}

Let $\mathrm{G}$ be a group acting on a finite set $\Omega$ of size $n$. If $x \in G$, define the index of $\mathrm{x}$ by ind $x=n-\operatorname{or} b(x)$ where $\operatorname{orb}(x)$ is the number of orbits of $\langle x\rangle$ on $\Omega$. Also Fix $x=\{\omega \in \Omega \mid x \omega=\omega\}, f(x)=\mid$ Fix $x \mid$. Furthermore, $\operatorname{orb}(x)=\frac{1}{d} \sum_{i=0}^{d-1} f\left(x^{i}\right)$ where $\mathrm{x}$ has order d.We discussed this method in detail in [2].

\section{Method two (Via Cycle Types)}

As we know that ind $x_{i}$ is the minimal number of 2-cycles needed to express $x_{i}$ as a product. We will label the fourteen nontrivial conjugacy classes of $S_{7}$ as ATLAS notation by:

Table 1. Non trivial conjugacy classes of $\boldsymbol{S}_{\mathbf{7}}$.

\begin{tabular}{|c|c|l|}
\hline Type & Conjugacy class & Ind \\
\hline 2A & $(1,2)^{S_{7}}$ & 1 \\
\hline 2B & $(1,2)(3,4)^{S_{7}}$ & 2 \\
\hline 2C & $(1,2)(3,4)(5,6)^{S_{7}}$ & 3 \\
\hline 3A & $(1,2,3)^{S_{7}}$ & 2 \\
\hline 3B & $(1,2,3)(4,5,6)^{S_{7}}$ & 4 \\
\hline 4A & $(1,2,3,4)^{S_{7}}$ & 3 \\
\hline 4B & $(1,2,3,4)(5,6)^{S_{7}}$ & 4 \\
\hline 5A & $(1,2,3,4,5)^{S_{7}}$ & 4 \\
\hline $6 \mathrm{~A}$ & $(1,2,3)(4,5)(6,7)^{S_{7}}$ & 4 \\
\hline 6B & $(1,2,3)(4,5)^{S_{7}}$ & 3 \\
\hline $6 \mathrm{C}$ & $(1,2,3,4,5,6)^{S_{7}}$ & 5 \\
\hline $7 \mathrm{~A}$ & $(1,2,3,4,5,6,7)^{S_{7}}$ & 6 \\
\hline 10A & $(1,2,3,4,5)(6,7)^{S_{7}}$ & 5 \\
\hline 12A & $(1,2,3,4)(5,6,7)^{S_{7}}$ & 5 \\
\hline
\end{tabular}




\section{CONNECTED COMPONENTS OF THE HURWITZ SPACE}

\section{Algorithm}

To achieve connected components of $\mathcal{H}_{r}^{i n}(G)$, we need to perform the following steps:

Step 1: Select the primitive group $S_{7}$ by using the GAP code [7] : Primitive Group $(7,7)$.

Step 2: Find all ramification types that satisfy equation (3) for given $S_{7}$, degree 7 and genus 0.

Step 3: Remove those types which have zero structure constant from the character table of $S_{7}$ via the following equation.

$$
n\left(C_{1}, \ldots, C_{k}\right)=\frac{\left|C_{1}\right|\left|C_{2}\right| \ldots\left|C_{k}\right|}{|G|} \sum_{\chi \in \operatorname{Irr}(G)} \frac{\chi\left(x_{1}\right) \chi\left(x_{2}\right) \ldots \chi\left(x_{k}\right)}{\chi(1)^{k-2}}
$$

With equation (5), we compute the number of k-tuples $\left(x_{1}, \ldots, x_{k}\right)$ of elements $x_{i}$ in the conjugacy class $C_{i}$ of a group $S_{7}$ such that $x_{1} x_{2} \ldots x_{k}=1$. In other words, we remove those types which don't satisfy equation (2).

Step 4: For the remaining types, that pass equation (1) which are called generating types.

Step 5: For the generating types, compute braid orbits by using MAPCLASS package.

Now we perform the above steps by using the program described in [12], but with a few modifications to it. That is, we remove the condition of affine type in that program. In this paper we only consider primitive groups.

\section{Results}

In this paper, we use the algorithm which is presented in section 3 to compute braid orbits on Nielsen class. An application of the algorithm is the classification of the primitive genus zero systems for $S_{7}$. That is, we find the connected components $\mathcal{H}_{\mathrm{r}}^{\text {in }}(\mathrm{C})$ of $\mathrm{S}_{7}$-curves $\mathrm{X}$, such that $\mathrm{g}\left(\mathrm{X} / \mathrm{S}_{-} 7\right)=0$. In our situation, the computation shows that there are exactly 1071 braid orbits of primitive genus 0 systems of degree 7 . The degree and the number of the branch points are given in Tables 2 and 3 . The detail of the Table 1 exists in [10].

Table 2. Primitive Genus Zero Systems: Number of Components.

\begin{tabular}{|c|l|l|l|l|l|l|l|}
\hline Degree & $\begin{array}{l}\text { \# Group Iso } \\
\text { types }\end{array}$ & \#RTs & $\begin{array}{l}\text { \# comp's } \\
\mathrm{r}=3\end{array}$ & $\begin{array}{l}\text { \# comp's } \\
\mathrm{r}=4\end{array}$ & $\begin{array}{l}\text { \# comp's } \\
\mathrm{r}=5\end{array}$ & $\begin{array}{l}\text { \# comp's } \\
\mathrm{r}=6\end{array}$ & $\begin{array}{l}\text { \# comp's } \\
\text { Total }\end{array}$ \\
\hline 7 & 5 & 154 & 179 & 61 & 67 & 10 & 317 \\
\hline
\end{tabular}

Table 3. Primitive Genus Zero Systems: Number of Components.

\begin{tabular}{|c|c|c|c|c|c|c|c|c|}
\hline Degree & $\begin{array}{c}\text { \# Group } \\
\text { Iso types }\end{array}$ & \#RTs & $\begin{array}{c}\text { \# comp's } \\
\mathrm{r}=3\end{array}$ & $\begin{array}{c}\text { \# comp's } \\
\mathrm{r}=4\end{array}$ & $\begin{array}{c}\text { \# comp's } \\
\mathrm{r}=5\end{array}$ & $\begin{array}{c}\text { \# comp's } \\
\mathrm{r}=6\end{array}$ & $\begin{array}{l}\text { \# comp's } \\
\mathrm{r}=7,8,9,10,11,12\end{array}$ & $\begin{array}{l}\text { \# comp's } \\
\text { Total }\end{array}$ \\
\hline 7 & 1 & 632 & 171 & 183 & 172 & 113 & $61,31,14,6,2,1$ & 754 \\
\hline
\end{tabular}

\section{Theorem 4.1}

Up to isomorphism, there exist exactly 6 primitive genus zero groups of degree seven. The corresponding primitive genus zero groups are enumerated in Table 4 and Tables 2-3.

\section{Lemma 4.2}

The Hurwitz spaces, $\mathcal{H}_{r}^{\text {in }}(C)$ are connected if $G=S_{7}$ and $r \geq 5$.

Proof. It follows from the fact that the Nielsen classes $\mathcal{N}(C)$ are the disjoint union of braid orbits but we have only one braid orbit for $S_{7}$ and $r \geq 5$. From Lemma 1.3, we obtain that the Hurwitz spaces $\mathcal{H}_{r}^{\text {in }}(C)$ are connected.

Lemma 4.3

The Hurwitz spaces, $\mathcal{H}_{r}^{\text {in }}(C)$ are disconnected if $G=S_{7}$ and $r \leq 4$.

Proof. Since we have at least two braid orbits for some type $C$ for $r \leq 4$ and $G=S_{7}$ and the Nielsen classes $\mathcal{N}(\mathrm{C})$ are the disjoint union of braid orbits. From Lemma 1.3, we obtain that the Hurwitz spaces $\mathcal{H}_{r}^{i n}(C)$ are disconnected. 
MOHAMMED SALIH, H.M.

Table 4. Primitive genus zero systems of $\boldsymbol{S}_{\mathbf{7}}$.

\begin{tabular}{|c|c|c|c|c|c|}
\hline $\begin{array}{l}\text { Ramification } \\
\text { Type }\end{array}$ & $\begin{array}{l}\text { Number of } \\
\text { orbits }\end{array}$ & $\begin{array}{l}\text { Length of } \\
\text { largest } \\
\text { orbit }\end{array}$ & $\begin{array}{l}\text { Ramification } \\
\text { Type }\end{array}$ & $\begin{array}{l}\text { Number of } \\
\text { orbits }\end{array}$ & $\begin{array}{l}\text { Length of } \\
\text { largest } \\
\text { orbit }\end{array}$ \\
\hline$(4 \mathrm{~A}, 5 \mathrm{~A}, 6 \mathrm{C})$ & 1 & 1 & $(3 \mathrm{~A}, 6 \mathrm{~B}, 4 \mathrm{~A}, 4 \mathrm{~B})$ & 1 & 141 \\
\hline$(4 \mathrm{~A}, 5 \mathrm{~A}, 10 \mathrm{~A})$ & 2 & 1 & $(3 \mathrm{~A}, 6 \mathrm{~B}, 4 \mathrm{~A}, 3 \mathrm{~B})$ & 1 & 64 \\
\hline$(4 \mathrm{~A}, 5 \mathrm{~A}, 12 \mathrm{~A})$ & 3 & 1 & $(3 \mathrm{~A}, 6 \mathrm{~B}, 4 \mathrm{~A}, 6 \mathrm{~A})$ & 1 & 69 \\
\hline$(4 \mathrm{~A}, 4 \mathrm{~B}, 6 \mathrm{C})$ & 4 & 1 & $(3 \mathrm{~A}, 6 \mathrm{~B}, 6 \mathrm{~B}, 5 \mathrm{~A})$ & 1 & 210 \\
\hline$(4 \mathrm{~A}, 4 \mathrm{~B}, 10 \mathrm{~A})$ & 4 & 1 & $(3 \mathrm{~A}, 6 \mathrm{~B}, 6 \mathrm{~B}, 4 \mathrm{~B})$ & 1 & 348 \\
\hline$(4 \mathrm{~A}, 4 \mathrm{~B}, 12 \mathrm{~A})$ & 4 & 1 & $(3 \mathrm{~A}, 6 \mathrm{~B}, 6 \mathrm{~B}, 3 \mathrm{~B})$ & 1 & 168 \\
\hline$(4 \mathrm{~A}, 4 \mathrm{~A}, 7 \mathrm{~A})$ & 1 & 1 & $(3 \mathrm{~A}, 6 \mathrm{~B}, 6 \mathrm{~B}, 6 \mathrm{~A})$ & 1 & 153 \\
\hline$(4 \mathrm{~A}, 3 \mathrm{~B}, 6 \mathrm{C})$ & 2 & 1 & $(3 \mathrm{~A}, 3 \mathrm{~A}, 4 \mathrm{~A}, 6 \mathrm{C})$ & 1 & 12 \\
\hline$(4 \mathrm{~A}, 3 \mathrm{~B}, 10 \mathrm{~A})$ & 3 & 1 & $(3 \mathrm{~A}, 3 \mathrm{~A}, 4 \mathrm{~A}, 10 \mathrm{~A})$ & 1 & 20 \\
\hline$(4 \mathrm{~A}, 3 \mathrm{~B}, 12 \mathrm{~A})$ & 1 & 1 & $(3 \mathrm{~A}, 3 \mathrm{~A}, 4 \mathrm{~A}, 12 \mathrm{~A})$ & 1 & 18 \\
\hline$(4 \mathrm{~A}, 6 \mathrm{~A}, 6 \mathrm{C})$ & 3 & 1 & $(3 \mathrm{~A}, 3 \mathrm{~A}, 6 \mathrm{~B}, 6 \mathrm{C})$ & 1 & 48 \\
\hline$(4 \mathrm{~A}, 6 \mathrm{~A}, 10 \mathrm{~A})$ & 1 & 1 & $(3 \mathrm{~A}, 3 \mathrm{~A}, 6 \mathrm{~B}, 10 \mathrm{~A})$ & 1 & 50 \\
\hline$(4 \mathrm{~A}, 6 \mathrm{~A}, 12 \mathrm{~A})$ & 2 & 1 & $(3 \mathrm{~A}, 3 \mathrm{~A}, 6 \mathrm{~B}, 12 \mathrm{~A})$ & 1 & 44 \\
\hline$(6 \mathrm{~B}, 5 \mathrm{~A}, 6 \mathrm{C})$ & 6 & 1 & $(3 \mathrm{~A}, 3 \mathrm{~A}, 2 \mathrm{C}, 6 \mathrm{C})$ & 1 & 20 \\
\hline$(6 \mathrm{~B}, 5 \mathrm{~A}, 10 \mathrm{~A})$ & 7 & 1 & $(3 \mathrm{~A}, 3 \mathrm{~A}, 2 \mathrm{C}, 10 \mathrm{~A})$ & 1 & 10 \\
\hline$(6 \mathrm{~B}, 5 \mathrm{~A}, 12 \mathrm{~A})$ & 5 & 1 & $(3 \mathrm{~A}, 3 \mathrm{~A}, 2 \mathrm{C}, 12 \mathrm{~A})$ & 1 & 16 \\
\hline$(6 \mathrm{~B}, 4 \mathrm{~B}, 6 \mathrm{C})$ & 12 & 1 & $(3 \mathrm{~A}, 2 \mathrm{C}, 4 \mathrm{~A}, 5 \mathrm{~A})$ & 1 & 30 \\
\hline$(6 \mathrm{~B}, 4 \mathrm{~B}, 10 \mathrm{~A})$ & 9 & 1 & $(3 \mathrm{~A}, 2 \mathrm{C}, 4 \mathrm{~A}, 4 \mathrm{~B})$ & 1 & 42 \\
\hline$(6 \mathrm{~B}, 4 \mathrm{~B}, 12 \mathrm{~A})$ & 9 & 1 & $(3 \mathrm{~A}, 2 \mathrm{C}, 4 \mathrm{~A}, 3 \mathrm{~B})$ & 1 & 27 \\
\hline$(6 \mathrm{~B}, 4 \mathrm{~A}, 7 \mathrm{~A})$ & 3 & 1 & $(3 \mathrm{~A}, 2 \mathrm{C}, 4 \mathrm{~A}, 6 \mathrm{~A})$ & 1 & 13 \\
\hline$(6 \mathrm{~B}, 3 \mathrm{~B}, 6 \mathrm{C})$ & 6 & 1 & $(3 \mathrm{~A}, 2 \mathrm{C}, 6 \mathrm{~B}, 5 \mathrm{~A})$ & 1 & 75 \\
\hline$(6 \mathrm{~B}, 3 \mathrm{~B}, 10 \mathrm{~A})$ & 4 & 1 & $(3 \mathrm{~A}, 2 \mathrm{C}, 6 \mathrm{~B}, 4 \mathrm{~B})$ & 1 & 105 \\
\hline$(6 \mathrm{~B}, 3 \mathrm{~B}, 12 \mathrm{~A})$ & 4 & 1 & $(3 \mathrm{~A}, 2 \mathrm{C}, 6 \mathrm{~B}, 3 \mathrm{~B})$ & 1 & 48 \\
\hline$(6 \mathrm{~B}, 6 \mathrm{~A}, 6 \mathrm{C})$ & 6 & 1 & $(3 \mathrm{~A}, 2 \mathrm{C}, 6 \mathrm{~B}, 6 \mathrm{~A})$ & 1 & 39 \\
\hline$(6 \mathrm{~B}, 6 \mathrm{~A}, 10 \mathrm{~A})$ & 4 & 1 & $(3 \mathrm{~A}, 2 \mathrm{C}, 2 \mathrm{C}, 5 \mathrm{~A})$ & 1 & 15 \\
\hline$(6 \mathrm{~B}, 6 \mathrm{~A}, 12 \mathrm{~A})$ & 3 & 1 & $(3 \mathrm{~A}, 2 \mathrm{C}, 2 \mathrm{C}, 4 \mathrm{~B})$ & 1 & 32 \\
\hline$(6 \mathrm{~B}, 6 \mathrm{~B}, 6 \mathrm{C})$ & 9 & 1 & $(3 \mathrm{~A}, 2 \mathrm{C}, 2 \mathrm{C}, 3 \mathrm{~B})$ & 1 & 13 \\
\hline$(3 \mathrm{~A}, 6 \mathrm{C}, 6 \mathrm{C})$ & 1 & 1 & $(3 \mathrm{~A}, 2 \mathrm{C}, 2 \mathrm{C}, 6 \mathrm{~A})$ & 1 & 11 \\
\hline$(3 \mathrm{~A}, 10 \mathrm{~A}, 6 \mathrm{C})$ & 2 & 1 & $(2 \mathrm{C}, 4 \mathrm{~A}, 4 \mathrm{~A}, 4 \mathrm{~A})$ & 1 & 32 \\
\hline$(3 \mathrm{~A}, 10 \mathrm{~A}, 10 \mathrm{~A})$ & 1 & 1 & $(2 \mathrm{C}, 6 \mathrm{~B}, 4 \mathrm{~A}, 4 \mathrm{~A})$ & 1 & 88 \\
\hline$(3 \mathrm{~A}, 12 \mathrm{~A}, 6 \mathrm{C})$ & 2 & 1 & $(2 \mathrm{C}, 6 \mathrm{~B}, 6 \mathrm{~B}, 4 \mathrm{~A})$ & 1 & 188 \\
\hline$(3 \mathrm{~A}, 12 \mathrm{~A}, 10 \mathrm{~A})$ & 2 & 1 & $(2 \mathrm{C}, 6 \mathrm{~B}, 6 \mathrm{~B}, 6 \mathrm{~B})$ & 2 & 102 \\
\hline$(3 \mathrm{~A}, 12 \mathrm{~A}, 12 \mathrm{~A})$ & 1 & 1 & $(2 \mathrm{C}, 2 \mathrm{C}, 4 \mathrm{~A}, 4 \mathrm{~A})$ & 1 & 16 \\
\hline$(2 \mathrm{C}, 5 \mathrm{~A}, 6 \mathrm{C})$ & 3 & 1 & $(2 \mathrm{C}, 2 \mathrm{C}, 6 \mathrm{~B}, 4 \mathrm{~A})$ & 1 & 56 \\
\hline$(2 \mathrm{C}, 5 \mathrm{~A}, 10 \mathrm{~A})$ & 1 & 1 & $(2 \mathrm{C}, 2 \mathrm{C}, 6 \mathrm{~B}, 6 \mathrm{~B})$ & 2 & 84 \\
\hline$(2 \mathrm{C}, 5 \mathrm{~A}, 12 \mathrm{~A})$ & 2 & 1 & $(2 \mathrm{C}, 2 \mathrm{C}, 2 \mathrm{C}, 4 \mathrm{~A})$ & 1 & 16 \\
\hline$(2 \mathrm{C}, 4 \mathrm{~B}, 6 \mathrm{C})$ & 4 & 1 & $(2 \mathrm{C}, 2 \mathrm{C}, 2 \mathrm{C}, 6 \mathrm{~B})$ & 1 & 36 \\
\hline$(2 \mathrm{C}, 4 \mathrm{~B}, 10 \mathrm{~A})$ & 3 & 1 & $(2 \mathrm{~B}, 4 \mathrm{~A}, 4 \mathrm{~A}, 5 \mathrm{~A})$ & 1 & 40 \\
\hline$(2 \mathrm{C}, 4 \mathrm{~B}, 12 \mathrm{~A})$ & 2 & 1 & $(2 \mathrm{~B}, 4 \mathrm{~A}, 4 \mathrm{~A}, 4 \mathrm{~B})$ & 1 & 96 \\
\hline$(2 \mathrm{C}, 4 \mathrm{~A}, 7 \mathrm{~A})$ & 1 & 1 & $(2 \mathrm{~B}, 4 \mathrm{~A}, 4 \mathrm{~A}, 3 \mathrm{~B})$ & 1 & 52 \\
\hline$(2 \mathrm{C}, 3 \mathrm{~B}, 10 \mathrm{~A})$ & 1 & 1 & $(2 \mathrm{~B}, 4 \mathrm{~A}, 4 \mathrm{~A}, 6 \mathrm{~A})$ & 1 & 44 \\
\hline$(2 \mathrm{C}, 3 \mathrm{~B}, 12 \mathrm{~A})$ & 1 & 1 & $(2 \mathrm{~B}, 6 \mathrm{~B}, 4 \mathrm{~A}, 5 \mathrm{~A})$ & 1 & 150 \\
\hline$(2 \mathrm{C}, 6 \mathrm{~A}, 6 \mathrm{C})$ & 1 & 1 & $(2 \mathrm{~B}, 6 \mathrm{~B}, 4 \mathrm{~A}, 4 \mathrm{~B})$ & 1 & 248 \\
\hline$(2 \mathrm{C}, 6 \mathrm{~A}, 10 \mathrm{~A})$ & 1 & 1 & $(2 \mathrm{~B}, 6 \mathrm{~B}, 4 \mathrm{~A}, 3 \mathrm{~B})$ & 1 & 118 \\
\hline$(2 \mathrm{C}, 6 \mathrm{~A}, 12 \mathrm{~A})$ & 1 & 1 & $(2 \mathrm{~B}, 6 \mathrm{~B}, 4 \mathrm{~A}, 6 \mathrm{~A})$ & 1 & 114 \\
\hline$(2 \mathrm{C}, 6 \mathrm{~B}, 7 \mathrm{~A})$ & 3 & 1 & $(2 \mathrm{~B}, 6 \mathrm{~B}, 6 \mathrm{~B}, 5 \mathrm{~A})$ & 1 & 395 \\
\hline$(2 \mathrm{~B}, 6 \mathrm{C}, 6 \mathrm{C})$ & 4 & 1 & $(2 \mathrm{~B}, 6 \mathrm{~B}, 6 \mathrm{~B}, 4 \mathrm{~B})$ & 1 & 620 \\
\hline$(2 \mathrm{~B}, 10 \mathrm{~A}, 6 \mathrm{C})$ & 3 & 1 & $(2 \mathrm{~B}, 6 \mathrm{~B}, 6 \mathrm{~B}, 3 \mathrm{~B})$ & 1 & 282 \\
\hline$(2 \mathrm{~B}, 10 \mathrm{~A}, 10 \mathrm{~A})$ & 3 & 1 & $(2 \mathrm{~B}, 6 \mathrm{~B}, 6 \mathrm{~B}, 6 \mathrm{~A})$ & 1 & 247 \\
\hline$(2 \mathrm{C}, 12 \mathrm{~A}, 6 \mathrm{C})$ & 3 & 1 & $(2 \mathrm{~B}, 3 \mathrm{~A}, 4 \mathrm{~A}, 6 \mathrm{C})$ & 1 & 33 \\
\hline$(2 \mathrm{C}, 12 \mathrm{~A}, 10 \mathrm{~A})$ & 2 & 1 & $(2 \mathrm{~B}, 3 \mathrm{~A}, 4 \mathrm{~A}, 10 \mathrm{~A})$ & 1 & 30 \\
\hline$(2 \mathrm{C}, 12 \mathrm{~A}, 12 \mathrm{~A})$ & 2 & 1 & $(2 \mathrm{~B}, 3 \mathrm{~A}, 4 \mathrm{~A}, 12 \mathrm{~A})$ & 1 & 34 \\
\hline$(2 \mathrm{~A}, 6 \mathrm{C}, 7 \mathrm{~A})$ & 1 & 1 & $(2 \mathrm{~B}, 3 \mathrm{~A}, 6 \mathrm{~B}, 6 \mathrm{C})$ & 1 & 102 \\
\hline$(2 \mathrm{~A}, 10 \mathrm{~A}, 7 \mathrm{~A})$ & 1 & 1 & $(2 \mathrm{~B}, 3 \mathrm{~A}, 6 \mathrm{~B}, 10 \mathrm{~A})$ & 1 & 85 \\
\hline$(2 \mathrm{~A}, 12 \mathrm{~A}, 7 \mathrm{~A})$ & 1 & 1 & $(2 \mathrm{~B}, 3 \mathrm{~A}, 6 \mathrm{~B}, 12 \mathrm{~A})$ & 1 & 75 \\
\hline$(4 \mathrm{~A}, 4 \mathrm{~A}, 4 \mathrm{~A}, 4 \mathrm{~A})$ & 1 & 16 & $(2 \mathrm{~B}, 3 \mathrm{~A}, 2 \mathrm{C}, 6 \mathrm{C})$ & 1 & 35 \\
\hline
\end{tabular}




\begin{tabular}{|c|c|c|c|c|c|}
\hline$(6 \mathrm{~B}, 4 \mathrm{~A}, 4 \mathrm{~A}, 4 \mathrm{~A})$ & 1 & 72 & $(2 \mathrm{~B}, 3 \mathrm{~A}, 2 \mathrm{C}, 10 \mathrm{~A})$ & 1 & 25 \\
\hline$(6 \mathrm{~B}, 6 \mathrm{~B}, 4 \mathrm{~A}, 4 \mathrm{~A})$ & 2 & 176 & $(2 \mathrm{~B}, 3 \mathrm{~A}, 2 \mathrm{C}, 12 \mathrm{~A})$ & 1 & 22 \\
\hline$(6 \mathrm{~B}, 6 \mathrm{~B}, 6 \mathrm{~B}, 4 \mathrm{~A})$ & 1 & 640 & $(2 \mathrm{~B}, 2 \mathrm{C}, 4 \mathrm{~A}, 5 \mathrm{~A})$ & 1 & 50 \\
\hline$(6 \mathrm{~B}, 6 \mathrm{~B}, 6 \mathrm{~B}, 6 \mathrm{~B})$ & 4 & 1008 & $(2 \mathrm{~B}, 2 \mathrm{C}, 4 \mathrm{~A}, 4 \mathrm{~B})$ & 1 & 80 \\
\hline$(3 \mathrm{~A}, 4 \mathrm{~A}, 4 \mathrm{~A}, 5 \mathrm{~A})$ & 1 & 15 & $(2 \mathrm{~B}, 2 \mathrm{C}, 4 \mathrm{~A}, 3 \mathrm{~B})$ & 1 & 36 \\
\hline$(3 \mathrm{~A}, 4 \mathrm{~A}, 4 \mathrm{~A}, 4 \mathrm{~B})$ & 1 & 44 & $(2 \mathrm{~B}, 2 \mathrm{C}, 4 \mathrm{~A}, 6 \mathrm{~A})$ & 1 & 28 \\
\hline$(3 \mathrm{~A}, 4 \mathrm{~A}, 4 \mathrm{~A}, 3 \mathrm{~B})$ & 1 & 23 & $(2 \mathrm{~B}, 2 \mathrm{C}, 6 \mathrm{~B}, 5 \mathrm{~A})$ & 1 & 125 \\
\hline$(3 \mathrm{~A}, 4 \mathrm{~A}, 4 \mathrm{~A}, 6 \mathrm{~A})$ & 1 & 34 & $(2 \mathrm{~B}, 2 \mathrm{C}, 6 \mathrm{~B}, 4 \mathrm{~B})$ & 1 & 176 \\
\hline$(3 \mathrm{~A}, 6 \mathrm{~B}, 4 \mathrm{~A}, 5 \mathrm{~A})$ & 1 & 65 & $(2 \mathrm{~B}, 2 \mathrm{C}, 6 \mathrm{~B}, 3 \mathrm{~B})$ & 1 & 84 \\
\hline$(2 \mathrm{~B}, 2 \mathrm{C}, 2 \mathrm{C}, 5 \mathrm{~A})$ & 1 & 35 & $(2 \mathrm{~B}, 2 \mathrm{C}, 6 \mathrm{~B}, 6 \mathrm{~A})$ & 1 & 65 \\
\hline$(2 \mathrm{~B}, 2 \mathrm{C}, 2 \mathrm{C}, 4 \mathrm{~B})$ & 1 & 48 & $(2 \mathrm{~A}, 2 \mathrm{C}, 3 \mathrm{~B}, 4 \mathrm{~B})$ & 1 & 24 \\
\hline$(2 \mathrm{~B}, 2 \mathrm{C}, 2 \mathrm{C}, 3 \mathrm{~B})$ & 1 & 22 & $(2 \mathrm{~A}, 2 \mathrm{C}, 3 \mathrm{~B}, 3 \mathrm{~B})$ & 1 & 12 \\
\hline$(2 \mathrm{~B}, 2 \mathrm{C}, 2 \mathrm{C}, 6 \mathrm{~A})$ & 1 & 18 & $(2 \mathrm{~A}, 2 \mathrm{C}, 6 \mathrm{~A}, 5 \mathrm{~A})$ & 1 & 11 \\
\hline$(2 \mathrm{~B}, 2 \mathrm{~B}, 4 \mathrm{~A}, 6 \mathrm{C})$ & 1 & 72 & $(2 \mathrm{~A}, 2 \mathrm{C}, 6 \mathrm{~A}, 4 \mathrm{~B})$ & 1 & 20 \\
\hline$(2 \mathrm{~B}, 2 \mathrm{~B}, 4 \mathrm{~A}, 10 \mathrm{~A})$ & 1 & 60 & $(2 \mathrm{~A}, 2 \mathrm{C}, 6 \mathrm{~A}, 3 \mathrm{~B})$ & 1 & 9 \\
\hline$(2 \mathrm{~B}, 2 \mathrm{~B}, 4 \mathrm{~A}, 12 \mathrm{~A})$ & 1 & 52 & $(2 \mathrm{~A}, 2 \mathrm{C}, 6 \mathrm{~A}, 6 \mathrm{~A})$ & 1 & 8 \\
\hline$(2 \mathrm{~B}, 2 \mathrm{~B}, 6 \mathrm{~B}, 6 \mathrm{C})$ & 1 & 198 & $(2 \mathrm{~A}, 2 \mathrm{C}, 6 \mathrm{~B}, 6 \mathrm{C})$ & 1 & 48 \\
\hline$(2 \mathrm{~B}, 2 \mathrm{~B}, 6 \mathrm{~B}, 10 \mathrm{~A})$ & 1 & 140 & $(2 \mathrm{~A}, 2 \mathrm{C}, 6 \mathrm{~B}, 10 \mathrm{~A})$ & 1 & 31 \\
\hline$(2 \mathrm{~B}, 2 \mathrm{~B}, 6 \mathrm{~B}, 12 \mathrm{~A})$ & 1 & 124 & $(2 \mathrm{~A}, 2 \mathrm{C}, 6 \mathrm{~B}, 12 \mathrm{~A})$ & 1 & 29 \\
\hline$(2 \mathrm{~B}, 2 \mathrm{~B}, 2 \mathrm{C}, 6 \mathrm{C})$ & 1 & 60 & $(2 \mathrm{~A}, 2 \mathrm{C}, 2 \mathrm{C}, 6 \mathrm{C})$ & 1 & 12 \\
\hline$(2 \mathrm{~B}, 2 \mathrm{~B}, 2 \mathrm{C}, 10 \mathrm{~A})$ & 1 & 40 & $(2 \mathrm{~A}, 2 \mathrm{C}, 2 \mathrm{C}, 10 \mathrm{~A})$ & 1 & 10 \\
\hline$(2 \mathrm{~B}, 2 \mathrm{~B}, 2 \mathrm{C}, 12 \mathrm{~A})$ & 1 & 36 & $(2 \mathrm{~A}, 2 \mathrm{C}, 2 \mathrm{C}, 12 \mathrm{~A})$ & 1 & 8 \\
\hline$(2 \mathrm{~A}, 4 \mathrm{~A}, 5 \mathrm{~A}, 5 \mathrm{~A})$ & 1 & 12 & $(2 \mathrm{~A}, 2 \mathrm{~B}, 5 \mathrm{~A}, 6 \mathrm{C})$ & 1 & 33 \\
\hline$(2 \mathrm{~A}, 4 \mathrm{~A}, 4 \mathrm{~B}, 5 \mathrm{~A})$ & 1 & 39 & $(2 \mathrm{~A}, 2 \mathrm{~B}, 5 \mathrm{~A}, 10 \mathrm{~A})$ & 1 & 28 \\
\hline$(2 \mathrm{~A}, 4 \mathrm{~A}, 4 \mathrm{~B}, 4 \mathrm{~B})$ & 1 & 80 & $(2 \mathrm{~A}, 2 \mathrm{~B}, 5 \mathrm{~A}, 12 \mathrm{~A})$ & 1 & 27 \\
\hline$(2 \mathrm{~A}, 4 \mathrm{~A}, 4 \mathrm{~A}, 6 \mathrm{C})$ & 1 & 12 & $(2 \mathrm{~A}, 2 \mathrm{~B}, 4 \mathrm{~B}, 6 \mathrm{C})$ & 1 & 60 \\
\hline$(2 \mathrm{~A}, 4 \mathrm{~A}, 4 \mathrm{~A}, 10 \mathrm{~A})$ & 1 & 16 & $(2 \mathrm{~A}, 2 \mathrm{~B}, 4 \mathrm{~B}, 10 \mathrm{~A})$ & 1 & 47 \\
\hline$(2 \mathrm{~A}, 4 \mathrm{~A}, 4 \mathrm{~A}, 12 \mathrm{~A})$ & 1 & 20 & $(2 \mathrm{~A}, 2 \mathrm{~B}, 4 \mathrm{~B}, 12 \mathrm{~A})$ & 1 & 42 \\
\hline$(2 \mathrm{~A}, 4 \mathrm{~A}, 3 \mathrm{~B}, 6 \mathrm{C})$ & 1 & 23 & $(2 \mathrm{~A}, 2 \mathrm{~B}, 4 \mathrm{~A}, 7 \mathrm{~A})$ & 1 & 14 \\
\hline$(2 \mathrm{~A}, 4 \mathrm{~A}, 3 \mathrm{~B}, 10 \mathrm{~A})$ & 1 & 38 & $(2 \mathrm{~A}, 2 \mathrm{~B}, 3 \mathrm{~B}, 6 \mathrm{C})$ & 1 & 30 \\
\hline$(2 \mathrm{~A}, 4 \mathrm{~A}, 3 \mathrm{~B}, 12 \mathrm{~A})$ & 1 & 12 & $(2 \mathrm{~A}, 2 \mathrm{~B}, 3 \mathrm{~B}, 10 \mathrm{~A})$ & 1 & 21 \\
\hline$(2 \mathrm{~A}, 4 \mathrm{~A}, 6 \mathrm{~A}, 5 \mathrm{~A})$ & 1 & 26 & $(2 \mathrm{~A}, 2 \mathrm{~B}, 3 \mathrm{~B}, 12 \mathrm{~A})$ & 1 & 19 \\
\hline$(2 \mathrm{~A}, 4 \mathrm{~A}, 6 \mathrm{~A}, 4 \mathrm{~B})$ & 1 & 34 & $(2 \mathrm{~A}, 2 \mathrm{~B}, 6 \mathrm{~A}, 6 \mathrm{C})$ & 1 & 27 \\
\hline$(2 \mathrm{~A}, 4 \mathrm{~A}, 6 \mathrm{~A}, 3 \mathrm{~B})$ & 1 & 21 & $(2 \mathrm{~A}, 2 \mathrm{~B}, 6 \mathrm{~A}, 10 \mathrm{~A})$ & 1 & 20 \\
\hline$(2 \mathrm{~A}, 4 \mathrm{~A}, 6 \mathrm{~A}, 6 \mathrm{~A})$ & 1 & 10 & $(2 \mathrm{~A}, 2 \mathrm{~B}, 6 \mathrm{~A}, 12 \mathrm{~A})$ & 1 & 17 \\
\hline$(2 \mathrm{~A}, 6 \mathrm{~B}, 5 \mathrm{~A}, 5 \mathrm{~A})$ & 1 & 62 & $(2 \mathrm{~A}, 2 \mathrm{~B}, 6 \mathrm{~B}, 7 \mathrm{~A})$ & 1 & 42 \\
\hline$(2 \mathrm{~A}, 6 \mathrm{~B}, 4 \mathrm{~B}, 5 \mathrm{~A})$ & 1 & 119 & $(2 \mathrm{~A}, 2 \mathrm{~B}, 2 \mathrm{C}, 7 \mathrm{~A})$ & 1 & 14 \\
\hline$(2 \mathrm{~A}, 6 \mathrm{~B}, 4 \mathrm{~B}, 4 \mathrm{~B})$ & 1 & 188 & $(2 \mathrm{~A}, 2 \mathrm{~A}, 6 \mathrm{C}, 6 \mathrm{C})$ & 1 & 12 \\
\hline$(2 \mathrm{~A}, 6 \mathrm{~B}, 4 \mathrm{~A}, 6 \mathrm{C})$ & 1 & 48 & $(2 \mathrm{~A}, 2 \mathrm{~A}, 10 \mathrm{~A}, 6 \mathrm{C})$ & 1 & 12 \\
\hline$(2 \mathrm{~A}, 6 \mathrm{~B}, 4 \mathrm{~A}, 10 \mathrm{~A})$ & 1 & 49 & $(2 \mathrm{~A}, 2 \mathrm{~A}, 10 \mathrm{~A}, 10 \mathrm{~A})$ & 1 & 10 \\
\hline$(2 \mathrm{~A}, 6 \mathrm{~B}, 4 \mathrm{~A}, 12 \mathrm{~A})$ & 1 & 41 & $(2 \mathrm{~A}, 2 \mathrm{~A}, 5 \mathrm{~A}, 7 \mathrm{~A})$ & 1 & 7 \\
\hline$(2 \mathrm{~A}, 6 \mathrm{~B}, 3 \mathrm{~B}, 5 \mathrm{~A})$ & 1 & 54 & $(2 \mathrm{~A}, 2 \mathrm{~A}, 12 \mathrm{~A}, 6 \mathrm{C})$ & 1 & 12 \\
\hline$(2 \mathrm{~A}, 6 \mathrm{~B}, 3 \mathrm{~B}, 4 \mathrm{~B})$ & 1 & 90 & $(2 \mathrm{~A}, 2 \mathrm{~A}, 12 \mathrm{~A}, 10 \mathrm{~A})$ & 1 & 10 \\
\hline$(2 \mathrm{~A}, 6 \mathrm{~B}, 3 \mathrm{~B}, 3 \mathrm{~B})$ & 1 & 42 & $(2 \mathrm{~A}, 2 \mathrm{~A}, 12 \mathrm{~A}, 12 \mathrm{~A})$ & 1 & 8 \\
\hline$(2 \mathrm{~A}, 6 \mathrm{~B}, 6 \mathrm{~A}, 5 \mathrm{~A})$ & 1 & 59 & $(2 \mathrm{~A}, 2 \mathrm{~A}, 4 \mathrm{~B}, 7 \mathrm{~A})$ & 1 & 14 \\
\hline$(2 \mathrm{~A}, 6 \mathrm{~B}, 6 \mathrm{~A}, 4 \mathrm{~B})$ & 1 & 77 & $(2 \mathrm{~A}, 2 \mathrm{~A}, 3 \mathrm{~B}, 7 \mathrm{~A})$ & 1 & 7 \\
\hline$(2 \mathrm{~A}, 6 \mathrm{~B}, 6 \mathrm{~A}, 3 \mathrm{~B})$ & 1 & 36 & $(2 \mathrm{~A}, 2 \mathrm{~A}, 6 \mathrm{~A}, 7 \mathrm{~A})$ & 1 & 7 \\
\hline$(2 \mathrm{~A}, 6 \mathrm{~B}, 6 \mathrm{~A}, 6 \mathrm{~A})$ & 1 & 28 & $(2 \mathrm{~B}, 2 \mathrm{~B}, 3 \mathrm{~A}, 6 \mathrm{~N}, 4 \mathrm{~A})$ & 1 & 2396 \\
\hline$(2 \mathrm{~A}, 6 \mathrm{~B}, 6 \mathrm{~B}, 6 \mathrm{C})$ & 1 & 144 & $(3 \mathrm{~A}, 3 \mathrm{~A}, 3 \mathrm{~A}, 4 \mathrm{~A}, 4 \mathrm{~A})$ & 1 & 163 \\
\hline$(2 \mathrm{~A}, 6 \mathrm{~B}, 6 \mathrm{~B}, 10 \mathrm{~A})$ & 1 & 112 & $(3 \mathrm{~A}, 3 \mathrm{~A}, 3 \mathrm{~A}, 6 \mathrm{~B}, 4 \mathrm{~A})$ & 1 & 606 \\
\hline$(2 \mathrm{~A}, 6 \mathrm{~B}, 6 \mathrm{~B}, 12 \mathrm{~A})$ & 1 & 100 & $(3 \mathrm{~A}, 3 \mathrm{~A}, 3 \mathrm{~A}, 6 \mathrm{~B}, 6 \mathrm{~B})$ & 1 & 1827 \\
\hline$(2 \mathrm{~A}, 2 \mathrm{~B}, 3 \mathrm{~A}, 2 \mathrm{C}, 3 \mathrm{~B})$ & 1 & 249 & $(2 \mathrm{~A}, 2 \mathrm{~A}, 2 \mathrm{~A}, 2 \mathrm{C}, 7 \mathrm{~A})$ & 1 & 49 \\
\hline$(2 \mathrm{~A}, 2 \mathrm{~B}, 3 \mathrm{~A}, 2 \mathrm{C}, 6 \mathrm{~A})$ & 1 & 197 & $(2 \mathrm{~A}, 2 \mathrm{~A}, 3 \mathrm{~A}, 3 \mathrm{~A}, 7 \mathrm{~A})$ & 1 & 49 \\
\hline$(2 \mathrm{~A}, 2 \mathrm{~B}, 2 \mathrm{C}, 4 \mathrm{~A}, 4 \mathrm{~A})$ & 1 & 400 & $(2 \mathrm{~A}, 2 \mathrm{~A}, 3 \mathrm{~A}, 2 \mathrm{C}, 6 \mathrm{C})$ & 1 & 132 \\
\hline$(2 \mathrm{~A}, 2 \mathrm{~B}, 2 \mathrm{C}, 6 \mathrm{~B}, 4 \mathrm{~A})$ & 1 & 976 & $(2 \mathrm{~A}, 2 \mathrm{~A}, 3 \mathrm{~A}, 2 \mathrm{C}, 10 \mathrm{~A})$ & 1 & 85 \\
\hline$(2 \mathrm{~A}, 2 \mathrm{~B}, 2 \mathrm{C}, 6 \mathrm{~B}, 6 \mathrm{~B})$ & 1 & 2256 & $(2 \mathrm{~A}, 2 \mathrm{~A}, 3 \mathrm{~A}, 2 \mathrm{C}, 12 \mathrm{~A})$ & 1 & 92 \\
\hline$(2 \mathrm{~A}, 2 \mathrm{~B}, 2 \mathrm{C}, 2 \mathrm{C}, 4 \mathrm{~A})$ & 1 & 276 & $(2 \mathrm{~A}, 2 \mathrm{~A}, 2 \mathrm{C}, 4 \mathrm{~A}, 5 \mathrm{~A})$ & 1 & 196 \\
\hline$(2 \mathrm{~A}, 2 \mathrm{~B}, 2 \mathrm{C}, 2 \mathrm{C}, 6 \mathrm{~B})$ & 1 & 612 & $(2 \mathrm{~A}, 2 \mathrm{~A}, 2 \mathrm{C}, 4 \mathrm{~A}, 4 \mathrm{~B})$ & 1 & 296 \\
\hline$(2 \mathrm{~A}, 2 \mathrm{~B}, 2 \mathrm{C}, 2 \mathrm{C}, 2 \mathrm{C})$ & 1 & 168 & $(2 \mathrm{~A}, 2 \mathrm{~A}, 2 \mathrm{C}, 4 \mathrm{~A}, 3 \mathrm{~B})$ & 1 & 156 \\
\hline$(2 \mathrm{~A}, 2 \mathrm{~B}, 2 \mathrm{~B}, 4 \mathrm{~A}, 5 \mathrm{~A})$ & 1 & 760 & $(2 \mathrm{~A}, 2 \mathrm{~A}, 2 \mathrm{C}, 4 \mathrm{~A}, 6 \mathrm{~A})$ & 1 & 100 \\
\hline$(2 \mathrm{~A}, 2 \mathrm{~B}, 2 \mathrm{~B}, 4 \mathrm{~A}, 4 \mathrm{~B})$ & 1 & 1264 & $(2 \mathrm{~A}, 2 \mathrm{~A}, 2 \mathrm{C}, 6 \mathrm{~B}, 5 \mathrm{~A})$ & 1 & 486 \\
\hline$(2 \mathrm{~A}, 2 \mathrm{~B}, 2 \mathrm{~B}, 4 \mathrm{~A}, 3 \mathrm{~B})$ & 1 & 600 & $(2 \mathrm{~A}, 2 \mathrm{~A}, 2 \mathrm{C}, 6 \mathrm{~B}, 4 \mathrm{~B})$ & 1 & 684 \\
\hline
\end{tabular}


MOHAMMED SALIH, H.M.

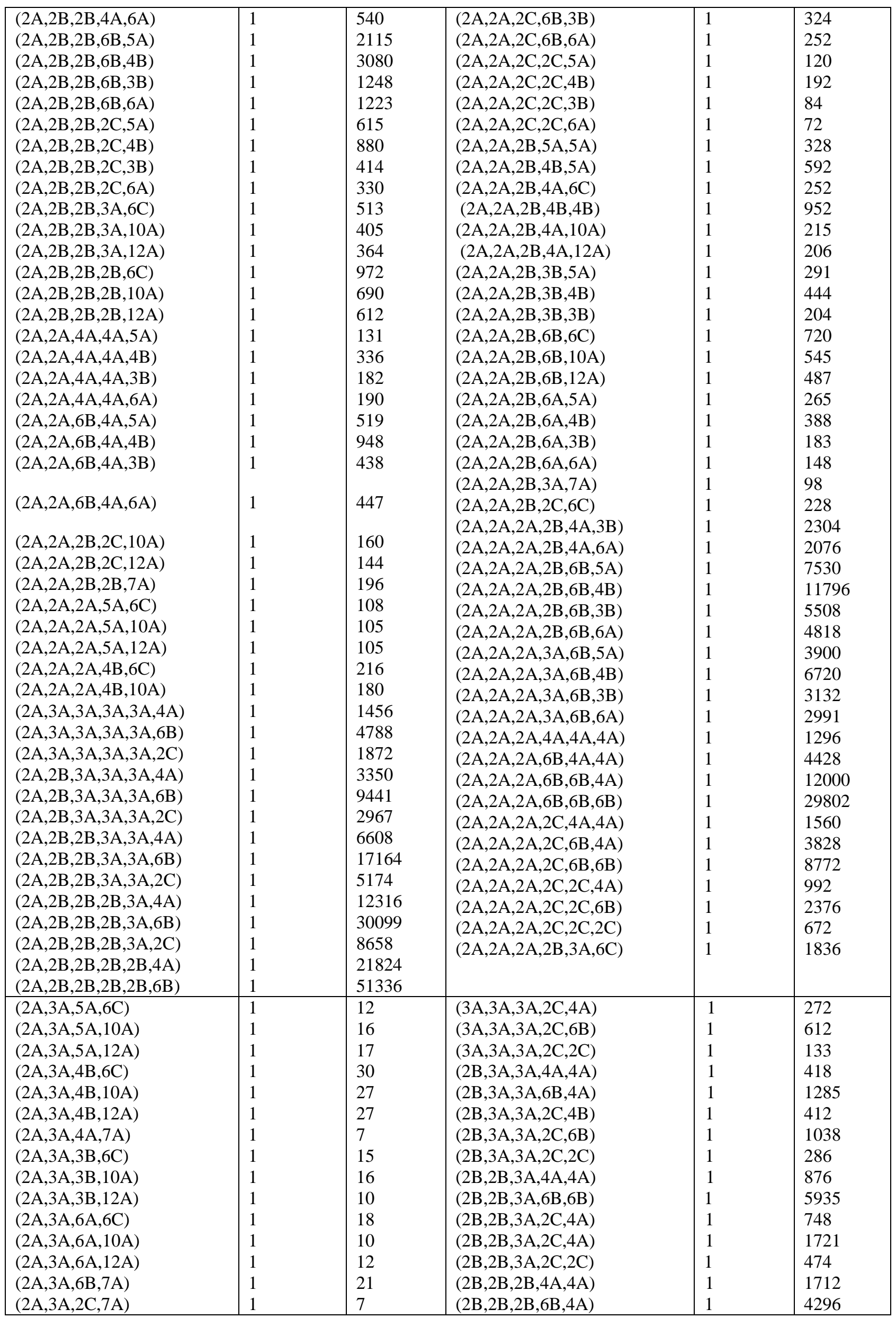




\begin{tabular}{|c|c|c|c|c|c|}
\hline$(2 \mathrm{~A}, 2 \mathrm{C}, 5 \mathrm{~A}, 5 \mathrm{~A})$ & 1 & 26 & $(2 \mathrm{~B}, 2 \mathrm{~B}, 2 \mathrm{~B}, 6 \mathrm{~B}, 6 \mathrm{~B})$ & 1 & 10128 \\
\hline$(2 \mathrm{~A}, 2 \mathrm{C}, 4 \mathrm{~B}, 5 \mathrm{~A})$ & 1 & 38 & $(2 \mathrm{~B}, 2 \mathrm{~B}, 2 \mathrm{~B}, 2 \mathrm{C}, 4 \mathrm{~A})$ & 1 & 1248 \\
\hline$(2 \mathrm{~A}, 2 \mathrm{C}, 4 \mathrm{~B}, 4 \mathrm{~B})$ & 1 & 56 & $(2 \mathrm{~B}, 2 \mathrm{~B}, 2 \mathrm{~B}, 2 \mathrm{C}, 6 \mathrm{~B})$ & 1 & 2880 \\
\hline$(2 \mathrm{~A}, 2 \mathrm{C}, 4 \mathrm{~A}, 6 \mathrm{C})$ & 1 & 20 & $(2 \mathrm{~B}, 2 \mathrm{~B}, 2 \mathrm{~B}, 2 \mathrm{C}, 2 \mathrm{C})$ & 1 & 748 \\
\hline$(2 \mathrm{~A}, 2 \mathrm{C}, 4 \mathrm{~A}, 10 \mathrm{~A})$ & 1 & 11 & $(2 \mathrm{~A}, 3 \mathrm{~A}, 4 \mathrm{~A}, 4 \mathrm{~A}, 4 \mathrm{~A})$ & 1 & 156 \\
\hline$(2 \mathrm{~A}, 2 \mathrm{C}, 4 \mathrm{~A}, 12 \mathrm{~A})$ & 1 & 14 & $(2 \mathrm{~A}, 3 \mathrm{~A}, 6 \mathrm{~B}, 4 \mathrm{~A}, 4 \mathrm{~A})$ & 1 & 589 \\
\hline$(2 \mathrm{~A}, 2 \mathrm{C}, 3 \mathrm{~B}, 5 \mathrm{~A})$ & 1 & 21 & $(2 \mathrm{~A}, 3 \mathrm{~A}, 6 \mathrm{~B}, 6 \mathrm{~B}, 4 \mathrm{~A})$ & 1 & 1782 \\
\hline$(2 \mathrm{~A}, 3 \mathrm{~A}, 3 \mathrm{~A}, 6 \mathrm{~B}, 5 \mathrm{~A})$ & 1 & 525 & $(2 \mathrm{~A}, 3 \mathrm{~A}, 6 \mathrm{~B}, 6 \mathrm{~B}, 6 \mathrm{~B})$ & 1 & 4455 \\
\hline$(2 \mathrm{~A}, 3 \mathrm{~A}, 3 \mathrm{~A}, 6 \mathrm{~B}, 4 \mathrm{~B})$ & 1 & 996 & $(2 \mathrm{~A}, 3 \mathrm{~A}, 3 \mathrm{~A}, 4 \mathrm{~A}, 5 \mathrm{~A})$ & 1 & 145 \\
\hline$(2 \mathrm{~A}, 3 \mathrm{~A}, 3 \mathrm{~A}, 6 \mathrm{~B}, 3 \mathrm{~B})$ & 1 & 462 & $(2 \mathrm{~A}, 3 \mathrm{~A}, 3 \mathrm{~A}, 4 \mathrm{~A}, 4 \mathrm{~B})$ & 1 & 350 \\
\hline$(2 \mathrm{~A}, 3 \mathrm{~A}, 3 \mathrm{~A}, 6 \mathrm{~B}, 6 \mathrm{~A})$ & 1 & 468 & $(2 \mathrm{~A}, 3 \mathrm{~A}, 3 \mathrm{~A}, 4 \mathrm{~A}, 3 \mathrm{~B})$ & 1 & 172 \\
\hline$(2 \mathrm{~A}, 3 \mathrm{~A}, 3 \mathrm{~A}, 3 \mathrm{~A}, 6 \mathrm{C})$ & 1 & 108 & $(2 \mathrm{~A}, 3 \mathrm{~A}, 3 \mathrm{~A}, 4 \mathrm{~A}, 6 \mathrm{~A})$ & 1 & 216 \\
\hline$(2 \mathrm{~A}, 3 \mathrm{~A}, 3 \mathrm{~A}, 3 \mathrm{~A}, 10 \mathrm{~A})$ & 1 & 135 & $(2 \mathrm{~A}, 2 \mathrm{~A}, 6 \mathrm{~B}, 6 \mathrm{~B}, 5 \mathrm{~A})$ & 1 & 1267 \\
\hline$(2 \mathrm{~A}, 3 \mathrm{~A}, 3 \mathrm{~A}, 3 \mathrm{~A}, 12 \mathrm{~A})$ & 1 & 120 & $(2 \mathrm{~A}, 2 \mathrm{~A}, 6 \mathrm{~B}, 6 \mathrm{~B}, 4 \mathrm{~B})$ & 1 & 2352 \\
\hline$(2 \mathrm{~A}, 3 \mathrm{~A}, 3 \mathrm{~A}, 2 \mathrm{C}, 5 \mathrm{~A})$ & 1 & 210 & $(2 \mathrm{~A}, 2 \mathrm{~A}, 6 \mathrm{~B}, 6 \mathrm{~B}, 3 \mathrm{~B})$ & 1 & 1098 \\
\hline$(2 \mathrm{~A}, 3 \mathrm{~A}, 3 \mathrm{~A}, 2 \mathrm{C}, 12 \mathrm{~A})$ & 1 & 300 & $(2 \mathrm{~A}, 2 \mathrm{~A}, 6 \mathrm{~B}, 6 \mathrm{~B}, 6 \mathrm{~A})$ & 1 & 975 \\
\hline$(2 \mathrm{~A}, 3 \mathrm{~A}, 3 \mathrm{~A}, 2 \mathrm{C}, 3 \mathrm{~B})$ & 1 & 168 & $(2 \mathrm{~A}, 2 \mathrm{~A}, 3 \mathrm{~A}, 6 \mathrm{~A}, 5 \mathrm{~A})$ & 1 & 170 \\
\hline$(2 \mathrm{~A}, 3 \mathrm{~A}, 3 \mathrm{~A}, 2 \mathrm{C}, 6 \mathrm{~A})$ & 1 & 105 & $(2 \mathrm{~A}, 2 \mathrm{~A}, 3 \mathrm{~A}, 6 \mathrm{~A}, 4 \mathrm{~B})$ & 1 & 236 \\
\hline$(2 \mathrm{~A}, 3 \mathrm{~A}, 2 \mathrm{C}, 4 \mathrm{~A}, 4 \mathrm{~A})$ & 1 & 238 & $(2 \mathrm{~A}, 2 \mathrm{~A}, 3 \mathrm{~A}, 6 \mathrm{~A}, 3 \mathrm{~B})$ & 1 & 126 \\
\hline$(2 \mathrm{~A}, 3 \mathrm{~A}, 2 \mathrm{C}, 6 \mathrm{~B}, 4 \mathrm{~A})$ & 1 & 587 & $(2 \mathrm{~A}, 2 \mathrm{~A}, 3 \mathrm{~A}, 6 \mathrm{~A}, 6 \mathrm{~A})$ & 1 & 82 \\
\hline$(2 \mathrm{~A}, 3 \mathrm{~A}, 2 \mathrm{C}, 6 \mathrm{~B}, 6 \mathrm{~B})$ & 1 & 1335 & $(2 \mathrm{~A}, 2 \mathrm{~A}, 3 \mathrm{~A}, 5 \mathrm{~A}, 5 \mathrm{~A})$ & 1 & 131 \\
\hline$(2 \mathrm{~A}, 3 \mathrm{~A}, 2 \mathrm{C}, 2 \mathrm{C}, 4 \mathrm{~A})$ & 1 & 136 & $(2 \mathrm{~A}, 2 \mathrm{~A}, 3 \mathrm{~A}, 4 \mathrm{~B}, 5 \mathrm{~A})$ & 1 & 302 \\
\hline$(2 \mathrm{~A}, 3 \mathrm{~A}, 2 \mathrm{C}, 2 \mathrm{C}, 6 \mathrm{~B})$ & 1 & 366 & $(2 \mathrm{~A}, 2 \mathrm{~A}, 3 \mathrm{~A}, 4 \mathrm{~B}, 4 \mathrm{~B})$ & 1 & 548 \\
\hline$(2 \mathrm{~A}, 3 \mathrm{~A}, 2 \mathrm{C}, 2 \mathrm{C}, 2 \mathrm{C})$ & 1 & 108 & $(2 \mathrm{~A}, 2 \mathrm{~A}, 3 \mathrm{~A}, 4 \mathrm{~A}, 6 \mathrm{C})$ & 1 & 82 \\
\hline$(2 \mathrm{~A}, 2 \mathrm{~B}, 4 \mathrm{~A}, 4 \mathrm{~A}, 4 \mathrm{~A})$ & 1 & 384 & $(2 \mathrm{~A}, 2 \mathrm{~A}, 3 \mathrm{~A}, 4 \mathrm{~A}, 10 \mathrm{~A})$ & 1 & 120 \\
\hline$(2 \mathrm{~A}, 2 \mathrm{~B}, 6 \mathrm{~B}, 4 \mathrm{~A}, 4 \mathrm{~A})$ & 1 & 1232 & $(2 \mathrm{~A}, 2 \mathrm{~A}, 3 \mathrm{~A}, 4 \mathrm{~A}, 12 \mathrm{~A})$ & 1 & 123 \\
\hline$(2 \mathrm{~A}, 2 \mathrm{~B}, 6 \mathrm{~B}, 6 \mathrm{~B}, 4 \mathrm{~A})$ & 1 & 3196 & $(2 \mathrm{~A}, 2 \mathrm{~A}, 3 \mathrm{~A}, 3 \mathrm{~B}, 5 \mathrm{~A})$ & 1 & 156 \\
\hline$(2 \mathrm{~A}, 2 \mathrm{~B}, 3 \mathrm{~A}, 4 \mathrm{~A}, 5 \mathrm{~A})$ & 1 & 355 & $(2 \mathrm{~A}, 2 \mathrm{~A}, 3 \mathrm{~A}, 3 \mathrm{~B}, 4 \mathrm{~B})$ & 1 & 264 \\
\hline$(2 \mathrm{~A}, 2 \mathrm{~B}, 6 \mathrm{~B}, 6 \mathrm{~B}, 6 \mathrm{~B})$ & 1 & 7752 & $(2 \mathrm{~A}, 2 \mathrm{~A}, 3 \mathrm{~A}, 3 \mathrm{~B}, 3 \mathrm{~B})$ & 1 & 105 \\
\hline$(2 \mathrm{~A}, 2 \mathrm{~B}, 3 \mathrm{~A}, 4 \mathrm{~A}, 4 \mathrm{~B})$ & 1 & 690 & $(2 \mathrm{~A}, 2 \mathrm{~A}, 2 \mathrm{~A}, 4 \mathrm{~B}, 12 \mathrm{~A})$ & 1 & 168 \\
\hline$(2 \mathrm{~A}, 2 \mathrm{~B}, 3 \mathrm{~A}, 4 \mathrm{~A}, 3 \mathrm{~B})$ & 1 & 349 & $(2 \mathrm{~A}, 2 \mathrm{~A}, 2 \mathrm{~A}, 4 \mathrm{~A}, 7 \mathrm{~A})$ & 1 & 49 \\
\hline$(2 \mathrm{~A}, 2 \mathrm{~B}, 3 \mathrm{~A}, 4 \mathrm{~A}, 6 \mathrm{~A})$ & 1 & 315 & $(2 \mathrm{~A}, 2 \mathrm{~A}, 2 \mathrm{~A}, 3 \mathrm{~B}, 6 \mathrm{C})$ & 1 & 108 \\
\hline$(2 \mathrm{~A}, 2 \mathrm{~B}, 3 \mathrm{~A}, 6 \mathrm{~B}, 5 \mathrm{~A})$ & 1 & 1085 & $(2 \mathrm{~A}, 2 \mathrm{~A}, 2 \mathrm{~A}, 3 \mathrm{~B}, 10 \mathrm{~A})$ & 1 & 90 \\
\hline$(2 \mathrm{~A}, 2 \mathrm{~B}, 3 \mathrm{~A}, 6 \mathrm{~B}, 4 \mathrm{~B})$ & 1 & 1763 & $(2 \mathrm{~A}, 2 \mathrm{~A}, 3 \mathrm{~A}, 6 \mathrm{~B}, 6 \mathrm{C})$ & 1 & 360 \\
\hline$(2 \mathrm{~A}, 2 \mathrm{~B}, 3 \mathrm{~A}, 6 \mathrm{~B}, 3 \mathrm{~B})$ & 1 & 828 & $(2 \mathrm{~A}, 2 \mathrm{~A}, 3 \mathrm{~A}, 6 \mathrm{~B}, 6 \mathrm{~A})$ & 1 & 325 \\
\hline$(2 \mathrm{~A}, 2 \mathrm{~B}, 3 \mathrm{~A}, 6 \mathrm{~B}, 6 \mathrm{~A})$ & 1 & 754 & $(2 \mathrm{~A}, 2 \mathrm{~A}, 3 \mathrm{~A}, 6 \mathrm{~B}, 12 \mathrm{~A})$ & 1 & 286 \\
\hline$(2 \mathrm{~A}, 2 \mathrm{~B}, 3 \mathrm{~A}, 3 \mathrm{~A}, 6 \mathrm{C})$ & 1 & 252 & $(2 \mathrm{~A}, 2 \mathrm{~A}, 2 \mathrm{~A}, 3 \mathrm{~B}, 12 \mathrm{~A})$ & 1 & 72 \\
\hline$(2 \mathrm{~A}, 2 \mathrm{~B}, 3 \mathrm{~A}, 3 \mathrm{~A}, 10 \mathrm{~A})$ & 1 & 220 & $(2 \mathrm{~A}, 2 \mathrm{~A}, 2 \mathrm{~A}, 6 \mathrm{~A}, 6 \mathrm{C})$ & 1 & 108 \\
\hline$(2 \mathrm{~A}, 2 \mathrm{~B}, 3 \mathrm{~A}, 3 \mathrm{~A}, 12 \mathrm{~A})$ & 1 & 220 & $(2 \mathrm{~A}, 2 \mathrm{~A}, 2 \mathrm{~A}, 6 \mathrm{~A}, 10 \mathrm{~A})$ & 1 & 75 \\
\hline$(2 \mathrm{~A}, 2 \mathrm{~B}, 3 \mathrm{~A}, 2 \mathrm{C}, 5 \mathrm{~A})$ & 1 & 350 & $(2 \mathrm{~A}, 2 \mathrm{~A}, 2 \mathrm{~A}, 6 \mathrm{~A}, 12 \mathrm{~A})$ & 1 & 7 \\
\hline$(2 \mathrm{~A}, 2 \mathrm{~B}, 3 \mathrm{~A}, 2 \mathrm{C}, 4 \mathrm{~B})$ & 1 & 536 & $(2 \mathrm{~A}, 2 \mathrm{~A}, 2 \mathrm{~A}, 6 \mathrm{~B}, 7 \mathrm{~A})$ & 1 & 147 \\
\hline$(2 \mathrm{~A}, 2 \mathrm{~B}, 2 \mathrm{~B}, 2 \mathrm{~B}, 2 \mathrm{~B}, 2 \mathrm{C})$ & 1 & 14448 & $(2 \mathrm{~A}, 2 \mathrm{~A}, 2 \mathrm{~A}, 2 \mathrm{~B}, 3 \mathrm{~A}, 10 \mathrm{~A})$ & 1 & 1500 \\
\hline$(2 \mathrm{~A}, 2 \mathrm{~A}, 3 \mathrm{~A}, 3 \mathrm{~A}, 4 \mathrm{~A}, 4 \mathrm{~A})$ & 1 & 1398 & $(2 \mathrm{~A}, 2 \mathrm{~A}, 2 \mathrm{~A}, 2 \mathrm{~B}, 3 \mathrm{~A}, 12 \mathrm{~A})$ & 1 & 1402 \\
\hline$(2 \mathrm{~A}, 2 \mathrm{~A}, 3 \mathrm{~A}, 3 \mathrm{~A}, 6 \mathrm{~B}, 4 \mathrm{~A})$ & 1 & 4588 & $(2 \mathrm{~A}, 2 \mathrm{~A}, 2 \mathrm{~A}, 2 \mathrm{~B}, 2 \mathrm{C}, 5 \mathrm{~A})$ & 1 & 2340 \\
\hline$(2 \mathrm{~A}, 2 \mathrm{~A}, 3 \mathrm{~A}, 3 \mathrm{~A}, 6 \mathrm{~B}, 6 \mathrm{~B})$ & 1 & 12600 & $(2 \mathrm{~A}, 2 \mathrm{~A}, 2 \mathrm{~A}, 2 \mathrm{~B}, 2 \mathrm{C}, 4 \mathrm{~B})$ & 1 & 3456 \\
\hline$(2 \mathrm{~A}, 2 \mathrm{~A}, 3 \mathrm{~A}, 3 \mathrm{~A}, 3 \mathrm{~A}, 5 \mathrm{~A})$ & 1 & 1260 & $(2 \mathrm{~A}, 2 \mathrm{~A}, 2 \mathrm{~A}, 2 \mathrm{~B}, 2 \mathrm{C}, 3 \mathrm{~B})$ & 1 & 1620 \\
\hline$(2 \mathrm{~A}, 2 \mathrm{~A}, 3 \mathrm{~A}, 3 \mathrm{~A}, 3 \mathrm{~A}, 4 \mathrm{~B})$ & 1 & 2640 & $(2 \mathrm{~A}, 2 \mathrm{~A}, 2 \mathrm{~A}, 2 \mathrm{~B}, 2 \mathrm{C}, 6 \mathrm{~A})$ & 1 & 1296 \\
\hline$(2 \mathrm{~A}, 2 \mathrm{~A}, 3 \mathrm{~A}, 3 \mathrm{~A}, 3 \mathrm{~A}, 3 \mathrm{~B})$ & 1 & 1260 & $(2 \mathrm{~A}, 2 \mathrm{~A}, 2 \mathrm{~A}, 2 \mathrm{~B}, 2 \mathrm{~B}, 6 \mathrm{C})$ & 1 & 3564 \\
\hline$(2 \mathrm{~A}, 2 \mathrm{~A}, 3 \mathrm{~A}, 3 \mathrm{~A}, 3 \mathrm{~A}, 6 \mathrm{~A})$ & 1 & 1422 & $(2 \mathrm{~A}, 2 \mathrm{~A}, 2 \mathrm{~A}, 2 \mathrm{~B}, 2 \mathrm{~B}, 10 \mathrm{~A})$ & 1 & 2650 \\
\hline$(2 \mathrm{~A}, 2 \mathrm{~A}, 2 \mathrm{~B}, 3 \mathrm{~A}, 3 \mathrm{~A}, 5 \mathrm{~A})$ & 1 & 2795 & $(2 \mathrm{~A}, 2 \mathrm{~A}, 2 \mathrm{~A}, 2 \mathrm{~B}, 2 \mathrm{~B}, 12 \mathrm{~A})$ & 1 & 2327 \\
\hline$(2 \mathrm{~A}, 2 \mathrm{~A}, 2 \mathrm{~B}, 3 \mathrm{~A}, 3 \mathrm{~A}, 4 \mathrm{~B})$ & 1 & 4952 & $(2 \mathrm{~A}, 2 \mathrm{~A}, 2 \mathrm{~A}, 2 \mathrm{~A}, 4 \mathrm{~A}, 6 \mathrm{C})$ & 1 & 864 \\
\hline$(2 \mathrm{~A}, 2 \mathrm{~A}, 2 \mathrm{~B}, 3 \mathrm{~A}, 3 \mathrm{~A}, 3 \mathrm{~B})$ & 1 & 2418 & $(2 \mathrm{~A}, 2 \mathrm{~A}, 2 \mathrm{~A}, 2 \mathrm{~A}, 4 \mathrm{~A}, 10 \mathrm{~A})$ & 1 & 800 \\
\hline$(2 \mathrm{~A}, 2 \mathrm{~A}, 2 \mathrm{~B}, 3 \mathrm{~A}, 3 \mathrm{~A}, 6 \mathrm{~A})$ & 1 & 2197 & $(2 \mathrm{~A}, 2 \mathrm{~A}, 2 \mathrm{~A}, 2 \mathrm{~A}, 4 \mathrm{~A}, 12 \mathrm{~A})$ & 1 & 784 \\
\hline$(2 \mathrm{~A}, 2 \mathrm{~A}, 2 \mathrm{~B}, 2 \mathrm{~B}, 3 \mathrm{~A}, 5 \mathrm{~A})$ & 1 & 5530 & $(2 \mathrm{~A}, 2 \mathrm{~A}, 2 \mathrm{~A}, 2 \mathrm{~A}, 5 \mathrm{~A}, 5 \mathrm{~A})$ & 1 & 1110 \\
\hline$(2 \mathrm{~A}, 2 \mathrm{~A}, 2 \mathrm{~B}, 2 \mathrm{~B}, 3 \mathrm{~A}, 4 \mathrm{~B})$ & 1 & 8896 & $(2 \mathrm{~A}, 2 \mathrm{~A}, 2 \mathrm{~A}, 2 \mathrm{~A}, 6 \mathrm{~B}, 6 \mathrm{C})$ & 1 & 2592 \\
\hline$(2 \mathrm{~A}, 2 \mathrm{~A}, 2 \mathrm{~B}, 2 \mathrm{~B}, 3 \mathrm{~A}, 3 \mathrm{~B})$ & 1 & 4191 & $(2 \mathrm{~A}, 2 \mathrm{~A}, 2 \mathrm{~A}, 2 \mathrm{~A}, 6 \mathrm{~B}, 10 \mathrm{~A})$ & 1 & 2100 \\
\hline$(2 \mathrm{~A}, 2 \mathrm{~A}, 2 \mathrm{~B}, 2 \mathrm{~B}, 3 \mathrm{~A}, 6 \mathrm{~A})$ & 1 & 3671 & $(2 \mathrm{~A}, 2 \mathrm{~A}, 2 \mathrm{~A}, 2 \mathrm{~A}, 6 \mathrm{~B}, 12 \mathrm{~A})$ & 1 & 1872 \\
\hline$(2 \mathrm{~A}, 2 \mathrm{~A}, 3 \mathrm{~A}, 3 \mathrm{~A}, 2 \mathrm{C}, 4 \mathrm{~A})$ & 1 & 1688 & $(2 \mathrm{~A}, 2 \mathrm{~A}, 2 \mathrm{~A}, 2 \mathrm{~A}, 3 \mathrm{~A}, 7 \mathrm{~A})$ & 1 & 343 \\
\hline$(2 \mathrm{~A}, 2 \mathrm{~A}, 3 \mathrm{~A}, 3 \mathrm{~A}, 2 \mathrm{C}, 6 \mathrm{~B})$ & 1 & 4044 & $(2 \mathrm{~A}, 2 \mathrm{~A}, 2 \mathrm{~A}, 2 \mathrm{~A}, 2 \mathrm{C}, 6 \mathrm{C})$ & 1 & 864 \\
\hline$(2 \mathrm{~A}, 2 \mathrm{~A}, 3 \mathrm{~A}, 3 \mathrm{~A}, 2 \mathrm{C}, 2 \mathrm{C})$ & 1 & 1020 & $(2 \mathrm{~A}, 2 \mathrm{~A}, 2 \mathrm{~A}, 2 \mathrm{~A}, 2 \mathrm{C}, 10 \mathrm{~A})$ & 1 & 600 \\
\hline$(2 \mathrm{~A}, 2 \mathrm{~A}, 2 \mathrm{~B}, 3 \mathrm{~A}, 4 \mathrm{~A}, 4 \mathrm{~A})$ & 1 & 3150 & $(2 \mathrm{~A}, 2 \mathrm{~A}, 2 \mathrm{~A}, 2 \mathrm{~A}, 2 \mathrm{C}, 12 \mathrm{~A})$ & 1 & 576 \\
\hline$(2 \mathrm{~A}, 2 \mathrm{~A}, 2 \mathrm{~B}, 3 \mathrm{~A}, 2 \mathrm{C}, 4 \mathrm{~A})$ & 1 & 2824 & $(2 \mathrm{~A}, 2 \mathrm{~A}, 2 \mathrm{~A}, 2 \mathrm{~A}, 4 \mathrm{~A}, 7 \mathrm{~A})$ & 1 & 686 \\
\hline
\end{tabular}


MOHAMMED SALIH, H.M.

\begin{tabular}{|c|c|c|c|c|c|}
\hline $\begin{array}{l}(2 \mathrm{~A}, 2 \mathrm{~A}, 2 \mathrm{~B}, 3 \mathrm{~A}, 2 \mathrm{C}, 6 \mathrm{~B}) \\
(2 \mathrm{~A}, 2 \mathrm{~A}, 2 \mathrm{~B}, 3 \mathrm{~A}, 2 \mathrm{C}, 2 \mathrm{C})\end{array}$ & $\begin{array}{l}1 \\
1\end{array}$ & $\begin{array}{l}6744 \\
1860\end{array}$ & $\begin{array}{l}(2 \mathrm{~A}, 2 \mathrm{~A}, 2 \mathrm{~A}, 2 \mathrm{~A}, 4 \mathrm{~B}, 5 \mathrm{~A}) \\
(2 \mathrm{~A}, 2 \mathrm{~A}, 2 \mathrm{~A}, 2 \mathrm{~A}, 4 \mathrm{~B}, 4 \mathrm{~B}) \\
(2 \mathrm{~A}, 2 \mathrm{~A}, 2 \mathrm{~A}, 2 \mathrm{~A}, 6 \mathrm{~A}, 5 \mathrm{~A})\end{array}$ & $\begin{array}{l}1 \\
1 \\
1\end{array}$ & $\begin{array}{l}2160 \\
3648 \\
1050\end{array}$ \\
\hline 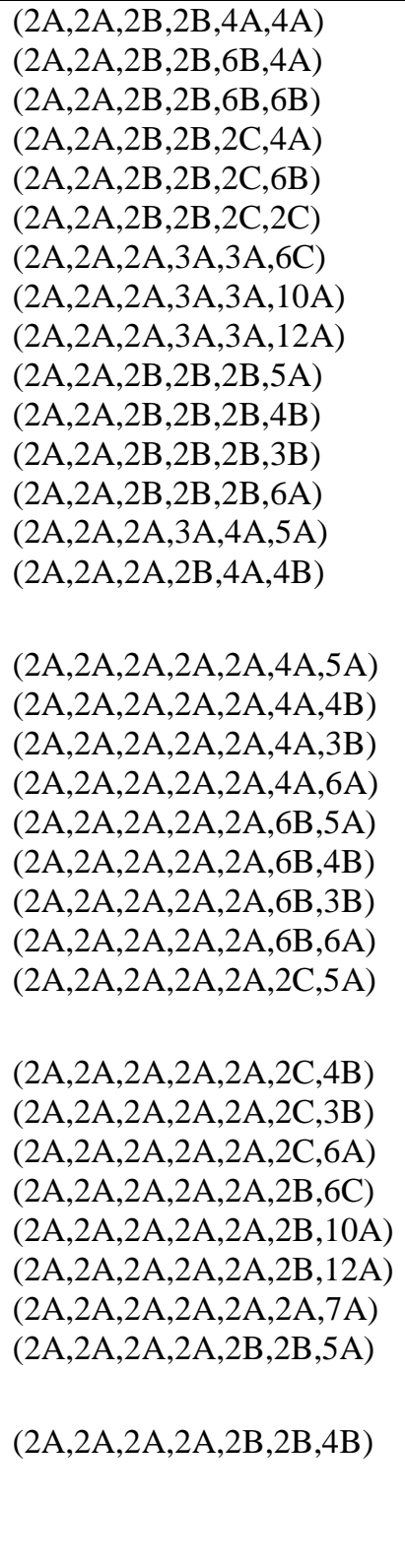 & $\begin{array}{l}1 \\
1 \\
1 \\
1 \\
1 \\
1 \\
1 \\
1 \\
1 \\
1 \\
1 \\
1 \\
1 \\
1 \\
1\end{array}$ & $\begin{array}{l}6320 \\
11628 \\
39288 \\
4880 \\
11268 \\
3080 \\
864 \\
850 \\
808 \\
10215 \\
15408 \\
7182 \\
6066 \\
1205 \\
4744 \\
\\
9500 \\
17600 \\
8640 \\
8160 \\
27750 \\
45120 \\
21060 \\
19080 \\
9000 \\
\\
13440 \\
8480 \\
5040 \\
12960 \\
10000 \\
9120 \\
2401 \\
38300 \\
\\
59264\end{array}$ & 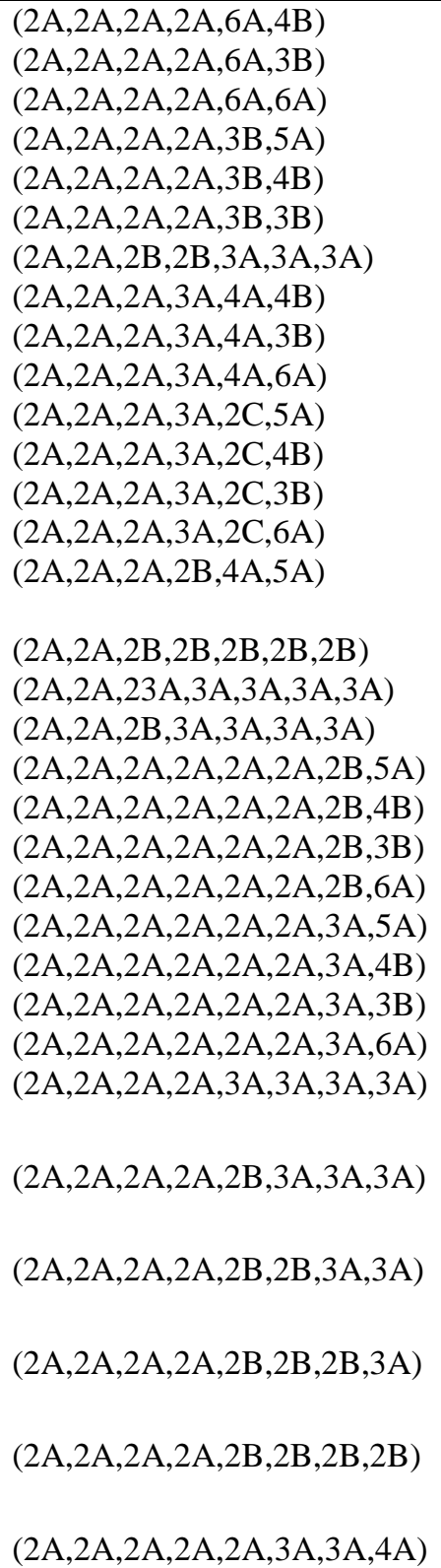 & $\begin{array}{l}1 \\
1 \\
1 \\
1 \\
1 \\
1 \\
1 \\
1 \\
1 \\
1 \\
1 \\
1 \\
1 \\
1 \\
1\end{array}$ & $\begin{array}{l}1536 \\
756 \\
756 \\
1080 \\
1728 \\
750 \\
48859 \\
2516 \\
1260 \\
1290 \\
1365 \\
2040 \\
1026 \\
744 \\
2710 \\
260848 \\
12207 \\
25860 \\
142500 \\
226560 \\
106920 \\
92880 \\
73125 \\
126720 \\
60750 \\
56160 \\
92880 \\
\\
183252 \\
336372 \\
593244 \\
1016904 \\
87840\end{array}$ \\
\hline
\end{tabular}


Table 5. Primitive genus zero systems of $\boldsymbol{S}_{\mathbf{7}}$.

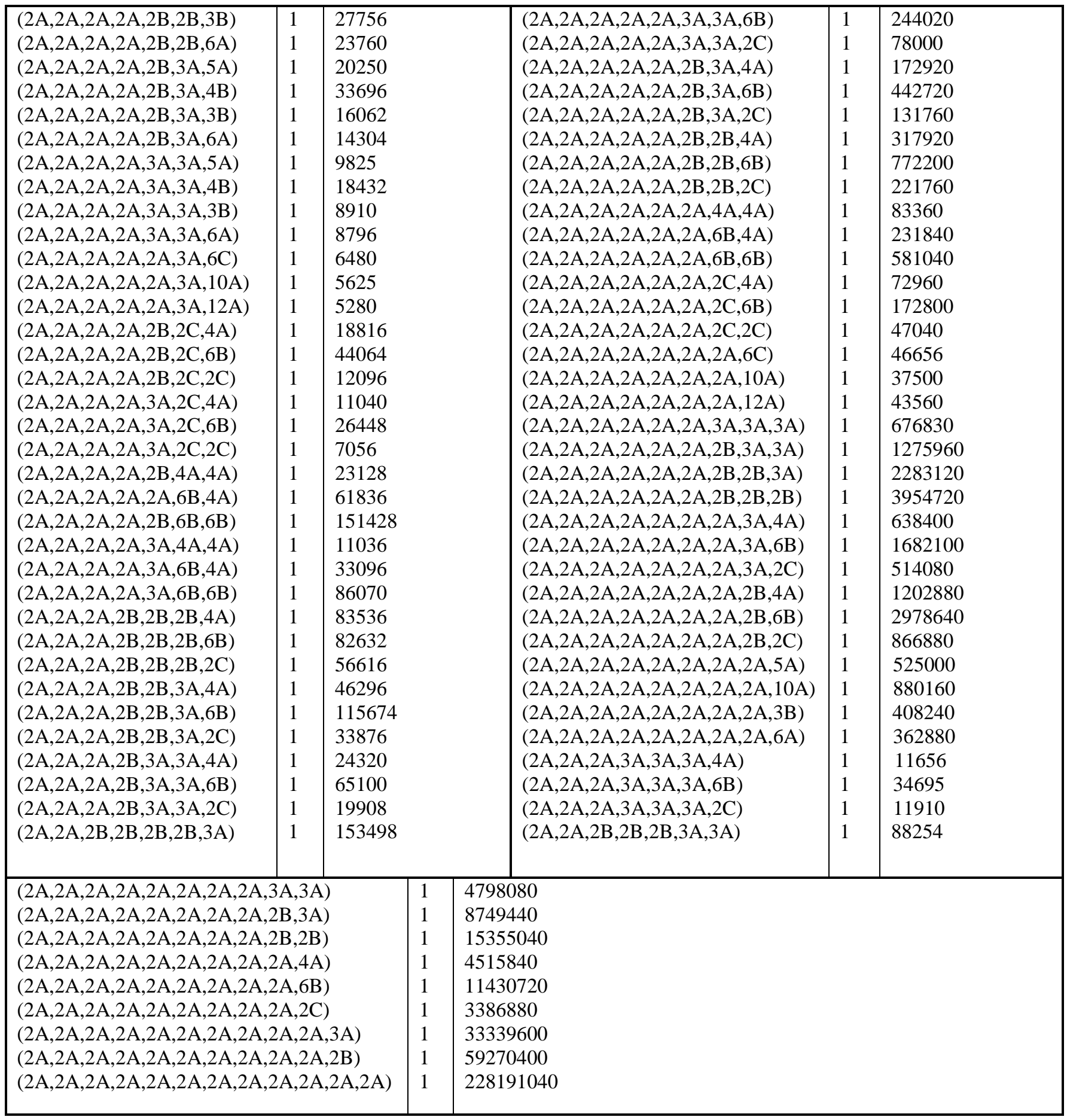

\section{Conclusion}

Here, we compute braid orbits on Nielsen class with the aid of the computer algebra system GAP and MAPCLASS package. A result of the algorithm is that it gives the complete classification of the symmetric group $S_{7}$ up to braid actions and diagonal conjugations. The computation shows that there are exactly 754 braid orbits of $S_{7}$. As a consequence of Lemma 1.3, we find the connected components $\mathcal{H}_{r}^{\text {in }}\left(S_{7}\right)$ of $S_{7}$-curves $X$, such that $g=0$. So we have 754 connected components $\mathcal{H}_{r}^{\text {in }}\left(S_{7}\right)$ of the symmetric group of degree seven.

\section{Acknowledgments}

The author would like thank the anonymous referees, whose comments and suggestions helped to improve the manuscript. 


\section{References}

1. Volklein, H. Groups as Galois groups An Introduction to Cambridge Studies in Advanced Mathematics, volume 53, Cambridge University Press, Cambridge, 1996.

2. Mohammed Salih, H. Finite Groups of Small Genus. 2014, Ph.D. Thesis, University of Birmingham.

3. Michael, D.F. and Volklein, H. The inverse Galois problem and rational points on moduli spaces. Mathematical Annual, 1991, 290(4), 771-800.

4. Frohardt D., Guralnick, R. and Magaard K. Genus 0 actions of groups of Lie rank 1. In Arithmetic fundamental groups and noncommutative algebra (Berkeley, CA, 1999), Proceeding Symposium in Pure Mathematics, 2002, 70, 449-483.

5. Frohardt, D. and Magaard, K. Composition factors of monodromy groups. American Mathematical Society, Providence, RI, Annals of Mathematics, 2001, 154(2), 327-345.

6. Frohardt, D., Guralnick, R. and Magaard, K. Primitive monodromy groups of genus at most two, Journal of Algebra, 2014, 417, 234-274.

7. The GAP Group. GAP-Groups, Algorithms, and Programming, Version 4.6.2, 2013.

8. Guralnick, R and John, G. Thompson. Finite groups of genus zero. Journal of Algebra, 1990, 131(1), 303-341.

9. Magaard, K., Shpectorov, S. and Wang, G. Generating sets of affine groups of low genus. In Computational algebraic and analytic geometry, Contemporary Mathematics, American Mathematical Society, Providence, RI, 2012, 572, 173-192.

10. Mohammed Salih, H. Connected components of $\mathcal{H}_{r}^{i n}(G)$. Sultan Qaboos University Journal for Science, 2017, 22(2), 106-113.

11. Neubauer, M. On solvable monodromy groups of fixed genus. 1989. Ph.D. Thesis, University of Southern California.

12. Guralnick, R. and John, G.T. Finite groups of genus zero. Journal of Algebra, 1990, 131(1), 303-341.

Received 16 January 2019

Accepted 12 June 2019 